\title{
Ingredients for Change: Food, Cooking and Mindfulness in Therapy
}

\author{
Lucy Rimalower* \\ Licensed Marriage and Family Therapist, USA
}

Submission: May 01, 2017; Published: May 04, 2017

*Corresponding author: Lucy Rimalower, M.A., LMFT, Los Angeles, CA, USA, Tel: +1(310)980-6329; Email: lucy@lucyrimalower.com

Abstract

Introducing psychotherapy clients to meditations that can be integrated into everyday activities such as cooking may help them to more easily cultivate a mindfulness practice. Given cognitive health benefits such as improved decision-making and creativity, a mindfulness practice may support them in attaining other personal goals.

Keywords: Mindfulness; Meditation; Psychotherapy; Cooking; Eating

\section{Opinion}

In the practice of psychotherapy, we are often faced with the challenge of helping clients bring useful ideas from therapy into their everyday lives. Consider a client who values caring for his body but struggles to exercise ora client who longs to be creative but struggles to sit down and write. Mindfulness may be an effective tool to bridge the gap between preference and lived experience, giving people a better chance of getting to the gym or the computer. That is, unless mindfulness becomes another unchecked box on a seemingly unattainable to-do list. Meditations and other exercises can be integrated into everyday activities like eating cereal or setting the dinner table, to improve the likelihood that a client actually practices mindfulness. Cooking and eating can provide an ideal platform on which to cultivate an organic marriage of habit and practice.

Mindfulness has gained phenomenal momentum in popular culture. It's used by schools, hospitals, corporate retreats, spas and, lest we forget its origins, religious organizations. "There's no doubt that as mindfulness has gone mainstream, plenty of people have used the technique to achieve peace of mind, greater selfawareness, perhaps even more compassion" writes David Gelles of the New York Times [1]. But with this ubiquity, mindfulness has become amorphous. There are formal practices of mindfulness meditation and codified evidence-based interventions used in mental health and other fields. Mindfulness can also be taken up as a more general mindset of interacting thoughtfully and nonjudgmentally with our environment, other people and ourselves.
Much of this popularity may be credited to Jon Kabat-Zinn who defines mindfulness as "paying attention in a particular way, on purpose, in the present moment, and non-judgmentally" [2]. In the 1970's, Kabat-Zinn [3,4] drew upon Buddhist principles intended to reduce suffering to create Mindfulness-Based Stress Reduction (MBSR) at the University of Massachusetts Medical School. This protocol allowed mindfulness to be used in a secular, scientific context. MBSR proved to be an effective resource for people suffering from chronic pain. Participants in Kabat-Zinn's programs also showed reduced anxiety [5], and improved brain and immune function [6]. Further, mindfulness has been shown to improve decision-making [7] as well as cognitive aspects of creativity including flexibility and originality in thinking [8].

These myriad cognitive, emotional and creative benefits have made mindfulness a compelling resource for mental health practitioners. But meditation competes for time with exercise, writing, and all the other items on the wellbeing wishlist. Not to mention the rest of our quotidian requirements. When a mindfulness practice is in place, the "sunk-cost bias" is mitigated Hafenbrack, Kinias, \& Barsade [7]. By moving our focus to the present moment, we may be freed from the narrative of failure that may result from thoughts about the past or even hopelessness for the future. This is to say, the decision to stay on the couch or get to the gym shifts away from the seemingly unrecoverable cost of what we didn't do yesterday or what we wish we could do tomorrow; rather, mindfulness allows us to suspend judgment to make the present moment ours and available. Which brings us back to the table. 
While clinicians should work within their scope of competence, there are simple exercises and meditations that can be introduced in session to cultivate mindfulness in existing routines. * Offering clients cooking and eating as a venue for meditation appeals to both convenience and creativity. We have to eat every day. We also have to prepare food, be that a gourmet meal or a Starbucks run. We can pay attention on purpose without judgment in any culinary scenario. Vago and Silbersweig [9] described two types of meditation: focused attention practices, in which a specific object or idea is maintained as the focus, and open monitoring practices, which more simply bring the mind back to the present moment without a particular object. While both are possible, food lends itself easily to focused attention practice, in part because of the rich sensory experience.

Consider Kabat-Zinn's classic exercise “Eating One Raisin: A First Taste of Meditation" [10]. Invite clients to observe a raisin (or perhaps a pistachio or other on-hand snack) through the five senses. Clients should describe the raisin in as much detail as possible through touch, sight, sound, smell, and taste, a practice usually reserved for wine-tasting. Such sensory inventories help clients slow down and get a detailed account of their own experience. This exercise takes just a few minutes and allows clients to practice focused attention. Clients can apply this to any food they choose.

More traditional meditations can also be used with foods, albeit non-traditionally. Tonglen, for example, as described by Pema Chödrön [11], comes from traditional Tibetan Buddhist practice in which you inhale while focusing on suffering in the world and exhale while sending out peaceful thoughts for healing and good will. Clients may choose an aromatic food or drink they are already planning to enjoy (ex: a cup of coffee, a slice of warm bread, a glass of wine). Inhale and exhale before each bite or sip.

There are also less formal ways to link food and mindfulness. Perhaps clients set an intention to purchase food that's grown ethically or safely. Maybe they want to pause to express gratitude for the meal before eating. Clients might concentrate on a more physical experience such as paying attention to their hunger and fullness. Or, they might opt to create a mindful chopping experience with vegetables, observing sounds, textures and symmetry. There are many ways to be playful with these ideas.
While more time spent on mindfulness practice yields more benefit [12], using food as a vehicle allows mindfulness into a client's life in small bites. Now back to the to-do list.

*Clinicians should be aware that further research is needed on populations for whom meditation may be contraindicated [13] and should consult with clients and their physicians about any potential health risks.

\section{References}

1. Gelles D (2016) The hidden price of mindfulness inc., New York Times, New York, USA, p. SR7.

2. Kabat-Zinn J (1994) Wherever you go, there you are: mindfulness meditation in everyday life. Hyperion, New York, USA.

3. Ludwig DS, Kabat-Zinn J (2008) Mindfulness in medicine. JAMA 300(11): 1350-1352.

4. Suzuki, Shunryū, Trudy Dixon, Huston Smith, Richard Baker (1970) Zen mind, beginner's mind. Weatherhill, New York, USA.

5. Kabat-Zinn J, Massion AO, Kristeller J, Peterson LG, Fletcher KE, et al. (1992) Effectiveness of a meditation-based stress reduction program in the treatment of anxiety disorders. Am J Psychiatry 149(7): 936-943.

6. Davidson RJ, Kabat-Zinn J, Schumacher J, Rosenkranz M, Muller D, et al. (2003) Alterations in brain and immune function produced by mindfulness meditation. Psychosom Med 65(4): 564-570.

7. Hafenbrack AC, Kinias Z, Barsade SG (2014) Debiasing the mind through meditation: mindfulness and the sunk-Cost Bias. Psychol Sci 25(2): 369-376.

8. Colzato LS, Ozturk A, Hommel B (2012) Meditate to create: the impact of focused-attention and open-monitoring training on convergent and divergent thinking. Front Psychol 3: 116.

9. Vago DR, Silbersweig DA (2012) Self-awareness, self-regulation, and self-transcendence (S-ART): A framework for understanding the neurobiological mechanisms of mindfulness. Front Hum Neurosci 6: 296.

10. Williams M, Teasdale J, Segal Z, Kabat-Zinn J (2007) The mindful way through depression: Freeing yourself from chronic unhappiness. Guilford Press, New York, USA, pp. 55-56.

11.Chödrön P, Ötro T (2001) Tonglen: The path of transformation. Vajradhatu Publications, Halifax, UK.

12. Luders E, Kurth F, Mayer E, Toga A, Narr K, et al. (2012) The unique brain anatomy of meditation practitioners: Alterations in cortical gyrification. Front Hum Neurosci 6: 34.

13. Hanley AW, Abell N, Osborn DS, Roehrig AD, Canto AI (2016). Mind the gaps: Are Conclusions about mindfulness Entirely conclusive? Journal of Counseling \& Development 94(1): 103-113.

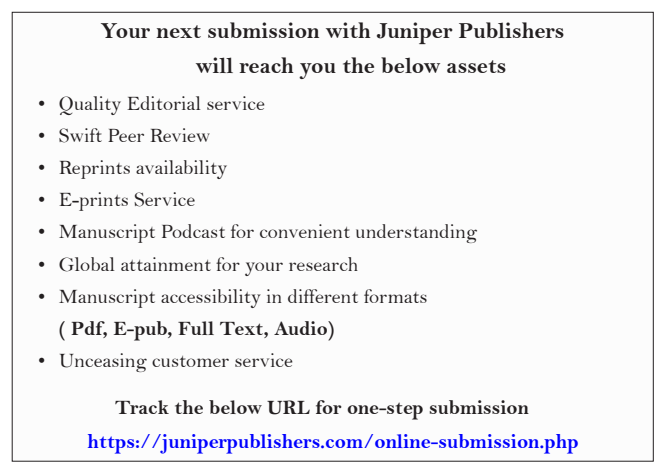

